\title{
ORTHOGONAL POLYNOMIALS WITH AUXILIARY CONDITIONS
}

\author{
$\mathrm{BY}$ \\ DUNHAM JACKSON
}

1. Introduction. Let $U_{1}(f), U_{2}(f), \cdots, U_{m}(f)$ be $m$ linear functionals, $m \geqq 1$, each defined for a class of functions $f$ including all polynomials in a single variable $x$. The characterization of a functional $U(f)$ as linear means here merely that if $f_{1}$ and $f_{2}$ are any two functions to which the operation applies, $U\left(c_{1} f_{1}+c_{2} f_{2}\right)=c_{1} U\left(f_{1}\right)+c_{2} U\left(f_{2}\right)$. This paper is concerned with sets of polynomials $p_{n}(x)$ orthogonal on an interval $(a, b)$, and satisfying the auxiliary conditions $U_{i}\left(p_{n}\right)=0$ for $i=1,2, \cdots, m$, and for each value of $n$. Two of the simplest special cases, one corresponding to the single condition $p_{n}(1)=p_{n}(-1)$ and the other to the condition $p_{n}(1)=-p_{n}(-1)$, have already been discussed elsewhere $\left({ }^{1}\right)$. It will be shown here that certain formal propositions with regard to such orthogonal systems can be stated with a considerable degree of generality, while the theory of convergence is carried appreciably beyond the stage previously attained.

2. Construction of the orthogonal system. If

$$
p_{n}(x)=a_{n 0}+a_{n 1} x+a_{n 2} x^{2}+\cdots+a_{n n} x^{n},
$$

it follows from the property of linearity that

$$
U_{i}\left(p_{n}\right)=\sum_{k=0}^{n} \gamma_{i k} a_{n k}, \quad \gamma_{i k}=U_{i}\left(x^{k}\right) .
$$

To the given set of auxiliary conditions there corresponds a matrix

$$
\begin{aligned}
& \begin{array}{llll}
\gamma_{10} & \gamma_{11} & \gamma_{12} & \cdots
\end{array} \\
& \begin{array}{llll}
\gamma_{20} & \gamma_{21} & \gamma_{22} & \cdots
\end{array} \\
& \gamma_{m 0} \quad \gamma_{m 1} \quad \gamma_{m 2} \cdots
\end{aligned}
$$

Presented to the Society, September 7, 1939; received by the editors January 11, 1940.

(1) See D. Jackson, $A$ new class of orthogonal polynomials, American Mathematical Monthly, vol. 46 (1939), pp. 493-497.

Since the present paper was written and since publication of the article in the Monthly, I have received through the kindness of Professor Mauro Picone a reprint of a paper by Wolfango Gröbner, Sistemi di polinomi ortogonali soddisfacenti a date condizioni, number 62 of the Pubblicazioni dell'Istituto per le Applicazioni del Calcolo, Consiglio Nazionale delle Ricerche, Rome, 1939, which also initiates a theory of orthogonal polynomials with linear homogeneous auxiliary conditions. That treatment and the one given here, however, diverge almost from the beginning as to methods and results to such an extent that there is very little duplication. 
with $m$ rows and infinitely many columns. Conversely, every such matrix, not consisting entirely of zeros, can be regarded as defining a set of $m$ (not necessarily independent) linear homogeneous conditions $U_{i}\left(p_{n}\right)=0$, significant for an arbitrary polynomial.

Let $r_{n}$ be the rank of the matrix of the first $n+1$ columns of (1), and let $r_{-1}=0$. If $r_{n}=r_{n-1}$, there exist polynomials

$$
P_{n}(x)=a_{0}+a_{1} x+a_{2} x^{2}+\cdots+a_{n} x^{n},
$$

with $a_{n} \neq 0$, satisfying the $m$ conditions $U_{i}\left(P_{n}\right)=0$. For if $a_{n}$ is taken equal to 1 , the relations to be satisfied by $a_{0}, \cdots, a_{n-1}$ are

$$
\sum_{k=0}^{n-1} \gamma_{i k} a_{k}=-\gamma_{i n}, \quad i=1,2, \cdots, m,
$$

and the condition $r_{n}=r_{n-1}$ is precisely the condition that the matrix of this system of equations have the same rank as the augmented matrix. If $r_{n} \neq r_{n-1}$, that is, if $r_{n}=r_{n-1}+1$, the equations are incompatible; the same is of course true if instead of 1 any other value different from zero is assigned to $a_{n}$, and there exists no polynomial of the $n$th degree with $a_{n} \neq 0$ satisfying the conditions.

As $n$ takes on the values $0,1,2, \cdots$, since $r_{n}$ can never decrease, can never increase by more than one unit at a time, and can never exceed $m$, there will be at most $m$ values of $n$ for which there is no polynomial satisfying the conditions. If $r_{n}$ never attains the value $m$, the $m$ conditions are linearly dependent; in the case of $m$ independent conditions there are $\left(^{2}\right)$ just $m$ exceptional values of $n$. It will be assumed henceforth that the conditions are independent.

Let polynomials satisfying the auxiliary conditions be constructed successively for all possible values of $n$, and let Schmidt's process be applied to these polynomials. It will be understood that the definition of orthogonality and normalization involves a weight function $\rho(x)$ which is non-negative, and positive on a set of positive measure on $(a, b)$. Let the orthogonal polynomials when normalized be denoted by $p_{n}(x)$, the subscript indicating the degree of the polynomial in each case. For convenience of notation, let $p_{n}(x) \equiv 0$ for the excluded values of $n$, and also for such negative values of $n$ as may enter in to any of the subsequent formulas.

Any polynomial of the $n$th degree satisfying the auxiliary conditions can be expressed linearly in terms of $p_{0}, p_{1}, \cdots, p_{n}$. For terms in $x^{n}, x^{n-1}, \ldots$ can be removed successively by subtraction of multiples of $p_{n}, p_{n-1}, \cdots$, leaving each time a polynomial which satisfies the conditions; when a degree is reached for which non-trivial polynomials satisfying the conditions do not

(2) See also Gröbner, loc. cit., p. 30, where the conclusion is stated with reference to a less general system of auxiliary conditions. 
exist, the leading coefficient in the corresponding remainder must already be zero.

3. Recursion formula and Christoffel-Darboux identity. The ordinary procedure for setting up a recursion formula does not apply without modification, for if a polynomial satisfying the auxiliary conditions is multiplied by $x$ the product does not satisfy the conditions in general. However, if each of the functionals $U_{i}(f)$ is expressible in terms of the values of $f$ at a finite number of points in the form

$$
U_{i}(f)=C_{i 1} f\left(y_{1}\right)+C_{i 2} f\left(y_{2}\right)+\cdots+C_{i v} f\left(y_{\nu}\right),
$$

where the $y$ 's are real, or else conjugate complex in pairs with corresponding conjugate complex coefficients, the conditions $U_{i}(f)=0$ are satisfied by any polynomial which vanishes at $y_{1}, y_{2}, \cdots, y_{\nu}$. (As a matter of notation, the list $y_{1}, \cdots, y_{\nu}$ is understood to include all the points that occur in any of the $U$ 's; some of the coefficients $C_{i j}$ may be zero.) If $q(x)$ is the product

$$
\left(x-y_{1}\right)\left(x-y_{2}\right) \cdots\left(x-y_{v}\right),
$$

or any polynomial divisible by this product (or, with trivial increase of generality but with a possible slight gain in simplicity or convenience, a constant plus any such polynomial $\left.\left({ }^{3}\right)\right), q(x) p_{n}(x)$ satisfies the conditions for each value of $n$, and is expressible linearly in terms of $p_{0}, \cdots, p_{n+\mu}$, where $\mu \geqq \nu$ is the degree of $q(x)$. By the property of orthogonality, the coefficient of $p_{k}(x)$ in this representation is zero for $k<n-\mu$, and the representation has the form

$$
q(x) p_{n}(x)=\sum_{k=n-\mu}^{n+\mu} c_{n k} p_{k}(x),
$$

with

$$
c_{n k}=\int_{a}^{b} \rho(x) q(x) p_{n}(x) p_{k}(x) d x .
$$

These formulas hold for all non-negative integral values of $n$ without exception, on the basis of the convention introduced above according to which $p_{k}(x)$ is identically zero when not defined otherwise.

From the recursion formula a Christoffel-Darboux identity can be derived in the usual way.

Similar reasoning is possible if $U_{i}(f)$ involves a finite number of derivatives at the points $y_{j}$. If $e_{j}$ is the order of the highest derivative involved at $y_{j}$, $q(x)$ as defined above is to be replaced by

$$
\prod_{j=1}^{\nu}\left(x-y_{j}\right)^{e_{j}+1}
$$

(3) E.g. in the earlier paper referred to, American Mathematical Monthly, loc. cit., the $y$ 's being the points $\pm 1, x^{2}$ was used as multiplier instead of $x^{2}-1$. 
or by a polynomial divisible by this product, or by a constant plus such a polynomial.

On the other hand, if there is just one auxiliary condition $U_{1}(f)=0$, where

$$
U_{1}(f)=\int_{-1}^{1} f(x) d x
$$

there is certainly no polynomial $q(x)$ (other than a constant) such that $q(x) p_{n}(x)$ satisfies the condition for all values of $n$. For that would require that $q(x)$ be orthogonal to every polynomial whose integral over $(-1,1)$ is zero, and so orthogonal to every Legendre polynomial of positive degree, and such a polynomial is a constant. There is no recursion formula which expresses $q(x) p_{n}(x)$ linearly in terms of the $p$ 's for all $n$, with a polynomial factor $q(x)$.

In the case of a single auxiliary condition $U_{1}(f)=0$, with

$$
U_{1}(f)=C_{1} f\left(y_{1}\right)+C_{2} f\left(y_{2}\right)+\cdots+C_{v} f\left(y_{v}\right),
$$

the one exceptional value of $n$ for which $p_{n}(x) \equiv 0$, the smallest value of $n$ for which $U_{1}\left(x^{n}\right) \neq 0$, cannot exceed $\nu-1$. For the equations $U_{1}\left(x^{k}\right)=0$, $k=0,1, \cdots, \nu-1$, constitute a set of linear equations for the $C$ 's, having for its determinant the nonvanishing Vandermonde determinant of the powers of the $y$ 's. That is to say, $U_{1}\left(x^{k}\right)$ cannot vanish for all these values of $k$ unless the $C$ 's are all zero.

If there are $m$ (linearly independent) conditions of the form (2), at least one $m$-rowed determinant of the first $\nu$ columns of (1) must be different from zero; $r_{n}=m$ for $n \geqq \nu-1$, and $p_{n}(x)$ is non-trivial for all values of $n \geqq \nu$. For if all the $m$-rowed determinants in the first $\nu$ columns were zero, the $m$ sets of quantities $U_{i}\left(x^{k}\right), k=0,1, \cdots, \nu-1$, would be linearly dependent; there would be numbers $b_{1}, \cdots, b_{m}$, not all zero, such that

$$
\sum_{i=1}^{m} b_{i}\left(C_{i 1} y_{1}^{k}+C_{i 2} y_{2}^{k}+\cdots+C_{i \nu} y_{\nu}^{k}\right)=0, \quad k=0,1, \cdots, \nu-1 ;
$$

that is,

$$
C_{1}^{\prime} y_{1}^{k}+C_{2}^{\prime} y_{2}^{k}+\cdots+C_{\nu}^{\prime} y_{\nu}^{k}=0, \quad C_{j}^{\prime}=\sum_{i=1}^{m} b_{i} C_{i j}
$$

By the argument of the preceding paragraph all the coefficients $C_{j}^{\prime}$ must vanish, which means that the $m$ sets of coefficients $C_{i 1}, \cdots, C_{i \nu}$ are linearly dependent.

4. Boundedness of the normalized polynomials; convergence. If $f(x)$ is an integrable function on $(a, b)$, it can be formally expanded in a series of the polynomials $p_{n}(x)$, the coefficients being determined in the usual way. When there is a Christoffel-Darboux identity, it can be used for the study of con-

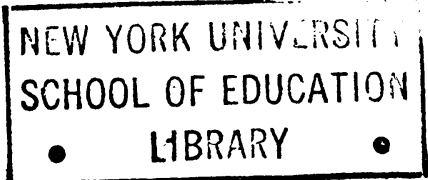


vergence in the same way as in connection with other orthogonal systems $\left(^{4}\right)$, if the polynomials $p_{n}(x)$ are bounded as $n$ becomes infinite, at the point where convergence is to be proved.

The discussion of boundedness here will be not so much a general theory as an exploration of the effectiveness of particular types of hypothesis leading to the property in question. The auxiliary conditions will in each case involve the values of the polynomials, or of the polynomials and their derivatives, at only a finite number of points, and even with this limitation will be rather highly specialized in form. The interval of orthogonality will for simplicity be taken as $(-1,1)$. The weight function, while open to subsequent generalization, will in the first instance be taken as unity.

Consider first the single condition $p_{n}\left(y_{1}\right)=p_{n}\left(-y_{1}\right)$. With $y_{1}=1$, this was treated in the earlier paper to which reference has been made. The condition is satisfied by any even polynomial, and by any polynomial which is divisible by $x^{2}-y_{1}^{2}$. For $n$ even let $p_{n}(x)$ denote the normalized Legendre polynomial of the $n$th degree. There is no polynomial of the first degree satisfying the auxiliary condition. For odd $n \geqq 3$ let $p_{n}(x)=\left(x^{2}-y_{1}^{2}\right) \pi_{n-2}(x)$, where $\pi_{k}(x)$ denotes the polynomial of the $k$ th degree in the orthonormal system corresponding to weight function $\left(x^{2}-y_{1}^{2}\right)^{2}$. For $n$ odd, $\pi_{n-2}(x)$ is an odd polynomial, since the weight function is even. Inasmuch as any odd polynomial is orthogonal to any even polynomial, the even and odd $p$ 's together constitute the desired orthogonal system. The normalized Legendre polynomials are uniformly bounded in any closed interval interior to $(-1,1)$. The same is true $\left(^{5}\right)$ of the polynomials $\left(x^{2}-y_{1}^{2}\right)^{2} \pi_{k}(x)$. Hence $p_{n}(x)$ is similarly bounded for odd as well as even $n$, if $y_{1}$ is not interior to the interval $(-1,1)$, and is uniformly bounded in the interval except near the points $\pm 1, \pm y_{1}$, if $y_{1}$ is between -1 and 1.

Suppose there are two conditions, $p_{n}\left(y_{1}\right)=p_{n}\left(-y_{1}\right), p_{n}\left(y_{2}\right)=p_{n}\left(-y_{2}\right)$. They are satisfied by any even polynomial, and by any polynomial divisible by $\left(x^{2}-y_{1}^{2}\right)\left(x^{2}-y_{2}^{2}\right)$. They are not satisfied by any polynomial of the first or third degree. The orthogonal system consists of the normalized even Legendre polynomials and the polynomials $\left(x^{2}-y_{1}^{2}\right)\left(x^{2}-y_{2}^{2}\right) \pi_{n-4}(x)$ with $n$ odd, where $\pi_{k}(x)$ denotes the general polynomial in the orthonormal system for weight $\left(x^{2}-y_{1}^{2}\right)^{2}\left(x^{2}-y_{2}^{2}\right)^{2}$. They are uniformly bounded throughout any closed interval interior to $(-1,1)$ and not containing any of the points $\pm y_{1}, \pm y_{2}$. The extension to an arbitrary number of conditions of the form $p_{n}\left(y_{j}\right)=p_{n}\left(-y_{j}\right)$ is obvious.

A set of conditions of the form $p_{n}\left(y_{j}\right)=-p_{n}\left(-y_{j}\right)$ leads to similar results. For a single condition $p_{n}\left(y_{1}\right)=-p_{n}\left(-y_{1}\right)$, the orthonormal system consists of

$\left(^{4}\right)$ See e.g. D. Jackson, Series of orthogonal polynomials, Annals of Mathematics, (2), vol.34 (1933), pp. 527-545; Orthogonal trigonometric sums, the same Annals, vol. 34 (1933), pp. 799814; A class of orthogonal functions on plane curves, the same Annals, vol. 40 (1939), pp. 521-532.

$\left.{ }^{5}\right)$ See e.g. D. Jackson, Series of orthogonal polynomials, loc. cit., pp. 534-535. 
the normalized odd Legendre polynomials and the polynomials $\left(x^{2}-y_{1}^{2}\right) \pi_{n-2}(x)$, $n=2,4, \cdots$, where $\pi_{0}, \pi_{2}, \cdots$ are the even orthonormal polynomials for $\left(x^{2}-y_{1}^{2}\right)^{2}$ as weight function.

With conditions of the form last mentioned, the ordinary proof of convergence, after the polynomials are known to be bounded, requires modification in one particular, because of the fact that the orthogonal system does not include a constant. Consider for definiteness the case of the single condition $p_{n}\left(y_{1}\right)=-p_{n}\left(-y_{1}\right)$. If $f(x)$ is a function developed in series of the $p$ 's, the partial sum of the series is given by

$$
s_{n}(x)=\int_{-1}^{1} f(t) K_{n}(t, x) d t, \quad K_{n}(t, x)=\sum_{k=1}^{n} p_{n}(t) p_{n}(x) .
$$

A polynomial of the $n$th or lower degree satisfying the auxiliary condition is reproduced by this formula exactly. For example,

$$
x=\int_{-1}^{1} t K_{n}(t, x) d t .
$$

If $f(x)$ can be represented in the form $x \phi(x)$, where $\phi(x)$ is a function of sufficient regularity, convergence can be treated by means of the formulas

$$
\begin{aligned}
& f(x)=x \phi(x)=\int_{-1}^{1} \phi(x) t K_{n}(t, x) d t \\
& s_{n}(x)-f(x)=\int_{-1}^{1}[\phi(t)-\phi(x)] t K_{n}(t, x) d t .
\end{aligned}
$$

The assumption that $f(x)$ can be represented in the form $x \phi(x)$ is no essential restriction, as far as convergence at other points than $x=0$ is concerned, for it can be seen as in other cases that convergence at a point depends only on the behavior of the function in the neighborhood of the point.

Occasion arises for a somewhat less simple treatment of the problem in connection, for example, with the auxiliary condition $p_{n}^{\prime}(1)=p_{n}^{\prime}(-1)$. This is satisfied by any odd polynomial; it is not satisfied by any polynomial of the second degree, but it is satisfied by a constant, or by any polynomial divisible by $\left(1-x^{2}\right)^{2}$. The even polynomials of the orthogonal system, however, do not consist merely of a constant and the polynomials $\left(1-x^{2}\right)^{2} q_{k}(x)$, where the $q$ 's are orthogonal for weight $\left(1-x^{2}\right)^{4}$; for example, $\left(1-x^{2}\right)^{2} q_{0}(x)$ is not orthogonal to a constant.

Let $p_{0}(x), p_{1}(x), p_{3}(x), p_{4}(x), \cdots$ be the orthonormal polynomials satisfying the auxiliary condition, and let $\xi_{0}(x), \xi_{1}(x), \xi_{2}(x), \cdots$ be the normalized Legendre polynomials. The odd $p$ 's are the odd $\xi$ 's. (It is readily seen, as in other problems having analogous features of symmetry, that the $p$ 's of even degree are even polynomials, and those of odd degree are odd.) For $n$ even let 
(3)

$$
\begin{aligned}
p_{n}(x) & =c_{n 0} \xi_{0}(x)+c_{n 1} \xi_{1}(x)+\cdots+c_{n n} \xi_{n}(x), \\
c_{n k} & =\int_{-1}^{1} p_{n}(x) \xi_{k}(x) d x .
\end{aligned}
$$

The polynomial $\xi_{k}(x)-\frac{1}{2} x^{2} \xi_{k}^{\prime}(1)$, with $k$ even, has a vanishing derivative for $x= \pm 1$, and satisfies the auxiliary condition. Hence $p_{n}(x)$ is orthogonal to it when $n>k$ :

$$
\int_{-1}^{1} p_{n}(x) \xi_{k}(x) d x-\xi_{k}^{\prime}(1) \int_{-1}^{1} \frac{1}{2} x^{2} p_{n}(x) d x=0, \quad k=0,2, \cdots, n-2,
$$

i.e., if the last integral is denoted by $g_{n}, c_{n k}=g_{n} \xi_{k}^{\prime}(1)$.

If $P_{k}(x)$ is the non-normalized Legendre polynomial, so that $\xi_{k}(x)$ $=[(2 k+1) / 2]^{1 / 2} P_{k}(x)$,

$$
P_{k}^{\prime}(1)=k(k+1) / 2, \quad \xi_{k}^{\prime}(1)=k(k+1)(2 k+1)^{1 / 2} / 2^{3 / 2} .
$$

Since $p_{n}(x)$ is normalized,

$$
1=\int_{-1}^{1}\left[p_{n}(x)\right]^{2} d x=\sum_{k=0}^{n} c_{n k}^{2}=c_{n n}^{2}+\frac{1}{8} g_{n}^{2} \sum_{k=0}^{n-2} k^{2}(k+1)^{2}(2 k+1),
$$

the sign $\sum^{\prime}$ indicating summation over even values of $k$. The sum by which $g_{n}^{2}$ is multiplied is of the order of magnitude of $n^{6}$, from which it follows that $g_{n}=O\left(1 / n^{3}\right)$.

Let

$$
\begin{gathered}
\alpha_{k}=[2 /(2 k+1)]^{1 / 2 \xi_{k}^{\prime}(1)=k(k+1) / 2} \\
S_{n}(x)=\sum_{k=0}^{n-2} \xi_{k}^{\prime}(1) \xi_{k}(x)=\sum_{k=0}^{n-2} \alpha_{k}[(2 k+1) / 2]^{1 / 2} \xi_{k}(x)=\sum_{k=0}^{n-2} \alpha_{k} \xi_{k}(1) \xi_{k}(x) .
\end{gathered}
$$

Let

$$
\sigma_{k}(x)=\sum_{j=0}^{k} \xi_{j}(1) \xi_{j}(x)
$$

for even $k$. Then ( $\alpha_{0}$ being zero)

$$
S_{n}(x)=\sum_{k=2}^{n-2} \alpha_{k}\left[\sigma_{k}(x)-\sigma_{k-2}(x)\right]=-\sum_{k=0}^{n-4}\left(\alpha_{k+2}-\alpha_{k}\right) \sigma_{k}(x)+\alpha_{n-2} \sigma_{n-2}(x) .
$$

Now, with summation extended over both odd and even values of $k$,

$$
\sum_{j=0}^{k} \xi_{j}(1) \xi_{j}(x)=\frac{k+1}{[(2 k+1)(2 k+3)]^{1 / 2}} \frac{\xi_{k+1}(1) \xi_{k}(\dot{x})-\xi_{k}(1) \xi_{k+1}(x)}{1-x},
$$

which, as $\xi_{k}(1)=O\left(k^{1 / 2}\right), \xi_{k}(x)=O(1)$, does not exceed a constant multiple 
of $k^{1 / 2}$ on a closed interval interior to $(-1,1)$. A similar statement holds if $x$ is replaced by $-x$, and consequently holds for the even and odd parts of the sum separately. In particular, $\left|\sigma_{k}(x)\right|=O\left(k^{1 / 2}\right)$ uniformly in any closed interval interior to $(-1,1)$. On the other hand, $\alpha_{k+2}-\alpha_{k}=O(k)$. So $\left|S_{n}(x)\right|=O\left(n^{5 / 2}\right)$.

The relation (3) may be written

$$
p_{n}(x)=c_{n n} \xi_{n}(x)+g_{n} S_{n}(x) .
$$

From the preceding paragraphs, $\left|g_{n} S_{n}(x)\right|=O\left(1 / n^{1 / 2}\right)$. By application of Schwarz's inequality to the integral defining the coefficient, $\left|c_{n n}\right| \leqq 1$. The polynomials $p_{n}(x)$ are uniformly bounded over any closed interval interior to $(-1,1)$.

An essentially similar problem is that associated with the condition $p_{n}^{\prime}(1)=-p_{n}^{\prime}(-1)$. The polynomials of even degree in the orthogonal system are Legendre polynomials. The requisite information about the coefficients in the representation of the odd polynomials of the system in terms of Legendre polynomials comes from the fact that $\xi_{k}(x)-x \xi_{k}^{\prime}(1)$ satisfies the auxiliary condition when $k$ is odd.

Considerations of the same sort are effective in connection with the unsymmetric condition $p_{n}(1)=h p_{n}(-1)$, where $h$ is an arbitrary constant $\neq \pm 1$. Here the orthogonal polynomials are neither even nor odd $\left(^{6}\right)$. In the representation

$$
p_{n}(x)=\sum_{k=0}^{n} c_{n k} \xi_{k}(x)
$$

the coefficients $c_{n k}$ for $k<n$ are determined in accordance with the fact that $\xi_{k}(x)-\mu \xi_{k}(1)$ satisfies the auxiliary condition with $\mu=(1+h) /(1-h)$ when $k$ is odd, and $\xi_{k}(x)-\xi_{k}(1)$ satisfies it when $k$ is even. Hence, for $k<n$, $c_{n k}=g_{n} \xi_{k}(1)$ or $\mu g_{n} \xi_{k}(1)$ according as $k$ is even or odd, with

Since

$$
g_{n}=\int_{-1}^{1} p_{n}(x) d x
$$

$$
\sum_{k=0}^{n} c_{n k}^{2}=1, \quad \xi_{k}(1)=[(2 k+1) / 2]^{1 / 2},
$$

it follows that $g_{n}=O(1 / n)$. And as was noted above, $\left|\sum \xi_{k}(1) \xi_{k}(x)\right|=O\left(n^{1 / 2}\right)$ in the interior of $(-1,1)$, whether the summation is extended over all subscripts from 0 to $n$, or over the even subscripts or the odd subscripts of the set separately. Hence the desired conclusion with regard to the boundedness of the p's.

The condition $p_{n}(1)=0$ leads merely to the set of polynomials

${ }^{(6)}$ For an explicit determination of these polynomials see Gröbner, loc. cit., pp. 46-47. 
$(x-1) \pi_{n-1}(x)$, where the $\pi$ 's are orthonormal for weight $(x-1)^{2}$. The condition $p_{n}^{\prime}(1)=0$ appears to be less trivial; boundedness of the $p$ 's can be proved by use of the observation that $\xi_{k}(x)-x \xi_{k}^{\prime}(1)$ has a vanishing derivative for $x=1$.

A primitive example of higher order is the condition $p_{n}^{\prime \prime}(1)=0$. It is satisfied by $\xi_{k}(x)-\frac{1}{2} x^{2} \xi_{k}^{\prime \prime}(1)$. So $p_{n}(x)$ is orthogonal to this expression for $k<n$, and if $p_{n}(x)=\sum_{k} c_{n k} \xi_{k}(x)$, then for $k<n$

$$
c_{n k}=g_{n} \xi_{k}^{\prime \prime}(1), \quad g_{n}=\int_{-1}^{1} \frac{1}{2} x^{2} p_{n}(x) d x .
$$

Since $\xi_{k}^{\prime \prime}(1)=\frac{1}{8}(k+2)(k+1) k(k-1) \xi_{k}(1)$, it follows by reasoning similar to that which has already been used that $g_{n}=O\left(1 / n^{5}\right),\left|\sum \xi_{k}^{\prime \prime}(1) \xi_{k}(x)\right|=O\left(n^{9 / 2}\right)$ in the interior of the interval, and the $p^{\prime}$ s are bounded as in other cases.

Consider next the pair of conditions $p_{n}(1)=p_{n}(-1), p_{n}^{\prime}(1)=p_{n}^{\prime}(-1)$. Because of the symmetry of the problem, the orthogonal polynomials are even or odd, and the even and odd sequences can be considered separately. When $n$ is even, the conditions are satisfied by $\xi_{n}(x)-\frac{1}{2} x^{2} \xi_{n}^{\prime}(1)$; when $n$ is odd they are satisfied by $\dot{\xi}_{n}(x)-x \xi_{n}(1)$. In each case it follows on the basis of calculations which have been presented already that the $p$ 's are bounded except near the ends of the interval.

As a final illustration, of somewhat more general character, suppose there is a single auxiliary condition $U_{1}\left(p_{n}\right)=0$ expressed in terms of the values of $p_{n}$ and an arbitrary finite number of its derivatives at the points \pm 1 . Let $x^{\lambda}$ be a power of $x$, for simplicity the lowest power, such that $U_{1}\left(x^{\lambda}\right) \neq 0$. Let

$$
\psi(x)=\xi_{k}(x)-A_{k} x^{\lambda} .
$$

The coefficient $A_{k}$ can be determined so that

$$
U_{1}(\psi)=U_{1}\left[\xi_{k}(x)\right]-A_{k} U_{1}\left(x^{\lambda}\right)=0 .
$$

When $A_{k}$ is thus determined, $p_{n}(x)$ is orthogonal to $\psi(x)$ if $n>k, n>\lambda$ :

$$
\int_{-1}^{1} p_{n}(x) \xi_{k}(x) d x=A_{k} g_{n}, \quad g_{n}=\int_{-1}^{1} x^{\lambda} p_{n}(x) d x .
$$

Since $\xi_{k}^{(h)}(-1)=(-1)^{k+h} \xi_{\mathfrak{k}}^{(h)}(1)$, there is a set of coefficients $a_{0}, a_{1}, \cdots, a_{\alpha}$, independent of $k$, with $a_{\alpha} \neq 0$, such that

$$
A_{k}=a_{0} \xi_{k}(1)+a_{1} \xi_{k}^{\prime}(1)+\cdots+a_{\alpha} \xi_{k}^{(\alpha)}(1)
$$

when $k$ is even, unless $A_{k}=0$ for all even values of $k$, and a set of coefficients $b_{0}, b_{1}, \cdots, b_{\beta}$, independent of $k$, with $b_{\beta} \neq 0$, such that

$$
A_{k}=b_{0} \xi_{k}(1)+b_{1} \xi_{k}^{\prime}(1)+\cdots+b_{\beta} \xi_{k}^{(\beta)}(1)
$$


when $k$ is odd, unless $A_{k}=0$ for all odd values of $k$; here $\alpha$ and $\beta$ are in general equal to the order $\gamma$ of the highest derivative occurring in $U_{1}\left(p_{n}\right)$, but one of them may in particular have a smaller value. At least one of the numbers $\alpha, \beta$ is certainly equal to $\gamma$, and at least one coefficient $a_{\gamma}$ or $b_{\gamma}$ is present with a value different from zero.

The quantity $\xi_{k}^{(\gamma)}(1)$ is of the order of magnitude of $k^{2 \gamma+(1 / 2)}$, and $\sum_{0}^{n-1} A_{k}^{2}$ is not less than a positive constant multiple of $n^{4 \gamma+2}$ when $n$ is sufficiently large. From the fact that $\sum_{k} c_{n k}^{2}=1$, if $p_{n}(x)=\sum c_{n k} \xi_{k}(x)$, it follows that $g_{n}=O\left(1 / n^{2 \gamma+1}\right)$. Since

$$
\left[\xi_{k+1}^{(\gamma)}(1) / \xi_{k+1}(1)\right]-\left[\xi_{k}^{(\gamma)}(1) / \xi_{k}(1)\right]=O\left(k^{2 \gamma-1}\right),
$$

it may be shown by the use of partial summation in conjunction with the Christoffel identity, in the manner previously indicated, together with inequalities obtained in the same way or, more simply, without resort to partial summation for the derivatives of lower order, that

$$
\left|\sum_{k=0}^{n-1} A_{k} \xi_{k}(x)\right|=O\left(n^{2 \gamma+(1 / 2)}\right)
$$

in the interior of $(-1,1)$. The $p$ 's are bounded as before.

It is apparent that the methods that have been used are capable of further extension. It is not so clear what the most general explicit formulation would be. On the other hand, it may be that some different method would lead to more general results at a single stroke.

The University of Minnesota, Minneapolis, MinN. 